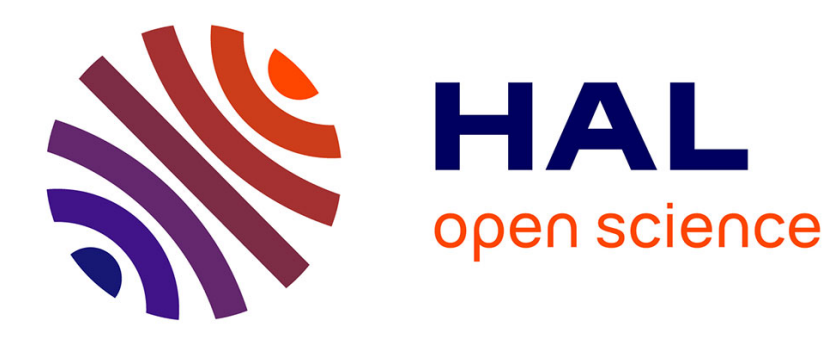

\title{
Sur l'invariabilité des constantes radioactives
}

Pierre Curie

\section{To cite this version:}

Pierre Curie. Sur l'invariabilité des constantes radioactives. J. Phys. Radium, 1929, 10 (9), pp.329336. 10.1051/jphysrad:01929001009032900 . jpa-00205393

\section{HAL Id: jpa-00205393 https://hal.science/jpa-00205393}

Submitted on 1 Jan 1929

HAL is a multi-disciplinary open access archive for the deposit and dissemination of scientific research documents, whether they are published or not. The documents may come from teaching and research institutions in France or abroad, or from public or private research centers.
L'archive ouverte pluridisciplinaire HAL, est destinée au dépôt et à la diffusion de documents scientifiques de niveau recherche, publiés ou non, émanant des établissements d'enseignement et de recherche français ou étrangers, des laboratoires publics ou privés. 


\title{
SUR L'INVARIABILITÉ DES CONSTANTES RADIOACTIVES
}

\author{
par $M^{\text {me }}$ P. CURIE
}

\begin{abstract}
Sommaire. - Cet article est un résumé concis de diverses expériences failes en vue de contrôler la régularité des transformations radioactives. Certaines de ces expériences étaient faites en vue d'influencer les constantes radioactives. D'autres ont été motivées par des anomalies qu'on a cru observer au cours de certains travaux et qui paraissaient impliquer une possibilité de variation de ces constantes. Dans l'ensemble, je n'ai pu, dans aucun cas, établir avec certitude un écart des lois généralement admises, bien que certains détails soient restés inexpliqués. L'objet de l'article est, en partie, d'attirer l'attention sur les difficultés qui se présentent dans l'étude de questions semblables.
\end{abstract}

Dans cet article j'ai décrit diverses expériences entreprises depuis plusieurs années en relation avec le problème d'invariabilité des constantes radioactives ${ }^{1}{ }^{1}$. Aucune variation certaine n'ayant été obtenue, l'exposé a été fait aussi bref que possible; il contient l'énumération des expériences et signale quelques difficuliés d'exécution et d'interprétation.

\section{Méthode d'observation.}

La méthode employée consistait dans la mesure du courant d'ionisation obtenu à l'aide d'une matière radioactive. Toute altération dans les lois normales établies pour la variation du courant avec le temps serait un indice d'altération d'une constante radioactive. Quand on désire obtenir le maximum de précision, on utilise une méthode de compensation, en mesurant la différence des courants d'ionisation produits dans des conditions semblables par deux échantillons d'une matière radioactive, dont l'un a subi une certaine action tandis que l'autre sert de témoin. L'avantage que présente ce mode opératoire a été exposé en détail dans une publication antérieure $\left({ }^{2}\right)$, où ont aussi été indiqués les procédés techniques qui permettent d'obtenir une haute précision sur les mesures quand on utilise le rayonnement $\gamma$. Dans le cas considéré alors, la comparaison portait sur deux ampoules contenant du radon, placées respectivement dans deux chambres d'ionisation identiques construites avec un soin particulier. Quand les deux ampoules sont tout à fait semblables, et contiennent du radon dilué, la mesure de la différence de leurs rayonnements : prouve que, pour chacune d'entre elles, la constante radioactive du radon ne peut subir pendant la durée des observations de variation atteignant 0,01 pour 100. Quand l'une des ampoules contient du radon dilué, 300 millicuries environ dans un volume de $10 \mathrm{~mm}^{3}$, et l'autre du radon très concentré, 300 millicuries dans un volume de $0,25 \mathrm{~mm}^{3}$, la constante radioactive du radon concentré conserve encore la valeur normale avec un écart inférieur à 0,0 po pour 100 , bien que le radon concentré soit soumis au rayonnement très intense .émis par lui-mème et par ses dérivés.

Il est impossible d'atteindre une précision semblable en utilisant les rayons $\alpha$ ou $\beta$, car les difficultés des mesures sont, en ce cas, plus grandes, surtout quand il s'agit de rayons $\alpha$. D'une part, les fluctuations radioactives du courant, à ionisation égale, sont beaucoup plus importantes pour les rayons $\alpha$ que pour les rayons $\gamma$; d'autre part, divers effets parasites se font d'autant plus sentir que les rayons sont moins pénétrants.

Soit $I$ l'intensité du courant d'ionisation. Une mesure dont la durée est $t$ correspond à une quantité d'électricité $Q=I t$ libérée dans la chambre d'ionisation par le passage d'un nombre $n$ de rayons dont la valeur moyenne est $Z=Q / q$, où $q$ est la quantité libérée par

(1) La bibliographie des nombreuses recherches faites dans le même but dans d'autres laboratoires et la discussion de ces travaux ne peuvent entrer dans le cadre de cet article.

(2) Mme P. Curie, Ann. de Phys., t 2 (1924), p. 405. 
rayon. La vraie valeur de $n$ pour un intervalle de temps $t$ étant $Z+\delta$, il existe pour un écart ò une certaine probabilité. On a $\Sigma \grave{o}=0$; d'autre part, la valeur la plus probable-

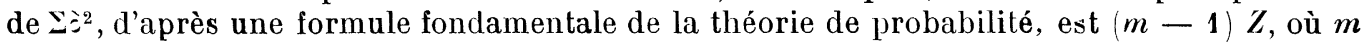
est le nombre d'intervalles de temps égaux utilisés pour une série de mesures. Si l'on mesure la différence $i$ de deux courants $I$ et $I^{\prime}$ auxquels correspondent les valeurs moyennes de $Z$ et $Z^{\prime}$ et les écarts individuels $\delta$ et $\delta^{\prime}$, la valeur moyenne de $\Sigma\left(\delta^{\prime}-\delta\right)$ est nulle, et la valeur la plus probable de $\Sigma\left(\delta^{\prime}-\delta\right)^{2}$ est la même que pour $\Sigma \delta^{2}+\Sigma \delta^{\prime 2}$, soit $(m-1)\left(Z+Z^{\prime}\right)$.

$\mathrm{Si} Z^{\prime}$ est peu différent de $Z$, la valeur la plus probable de l'écart quadratique $\operatorname{sur}\left(Z^{\prime}-Z\right)$ est double de celle relative à chacune de ces quantités séparément.

La fluctuation sur la mesure des courants $I$ et $I^{\prime}$ et de leur diffẻrence $i$ peut être influencée par l'appareil électrométrique qui n'est pas un indicateur instantané de courant $I$ ou de quantité $Q$. Il paraît cependant probable que l'inertie de cet appareil' n'apporte pas de perturbation notable quand les mesures sont faites par compensation à l'aide d'une quantité d'électricité bien déterminée produite en un intervalle de temps de durée suffisante.

Il n'est pas nécessaire de tenir compte d'une fluctuation sur $q$, car l'effet correspondant s'éliminerait dans l'addition des quantités d'électricité libérées par le grand nombre de rayons utilisés pour une mesure. En désignant par $\Delta$ l'écart sur la quantité $Q=Z q$, on a, par conséquent, $\Delta=q \delta$, et la valeur la plus probable de $\Sigma \Delta^{2}$ est $(m-1) q^{2} Z=(m-1) Q q$. On voit qu'à quantité $Q$ égale mise en liberté dans le même temps, c'est-à-dire, à courant $I$ égal, l'importance des fluctuations est proportionelle au pouvoir ionisant d'un rayon; elle est donc beaucoup plus grande pour les rayons $\alpha$ que pour les rayons $\gamma$.

I. Exposition d'un tube de radon au rayonnement pénêtrant du radium. L'expérience consiste à comparer le rayonnement $\gamma$ de deux tubes de verre scellés contenant du radon, dont l'un seulement est exposé au rayonnement dans l'intervalle des mesures. On a employé l'appareil qui avait servi pour l'étude de la décroissance du radon concentré et les mesures ont été conduites et interprétées de la même manière. Après l'exposition l'ampoule pouvait être remise en place avec une exactitude rigoureuse. Les deux ampoules contenaient presque exactement la même quantitě de radon égale à $\mathbf{1 5}$, ¿̈̉ millicuries. L'exposition a consisté à introduire l'une d'elles dans un tube de verre mince, entouré de six tubes de verre scellés dont chacun contenait $100 \mathrm{mgr}$ de radium et était placé dans un tube de laiton à parois mincess. La durée de l'exposition, 118 heures, a été prise voisine de la vie moyenne du radon. $\mathrm{Si}$, en effet, on se place dans l'hypothèse où un rayonnement constant produirait une variation fixe relative $\triangleq$ de la constante radioactive $\lambda$ pour le courant $I^{\prime}$, et si à l'origine $I_{0}=I_{0}^{\prime}$, la différence $i$ serait donněe par la formule

$$
i=I_{0} \mathrm{e}^{-\lambda t}-I_{0} \mathrm{e}^{-\lambda(1+\varepsilon) t}
$$

Cette quantité, nulle pour $t=0$ et $t=\infty$, passe par un maximum pour $t=1 \lambda$.

La valeur initiale du courant $l$ était environ $1,2 \mathrm{E}$. S.; la valeur du rapport $i / \boldsymbol{I}$ était 92,4 (rapport d'amplification). Les points représentatifs de Log $i$ en fonction du temps, avant et après l'exposition, se placent sur une droite dont la pente diffère de 1/300 à peine de la pente normale. La variation correspondante pour la pente de la droite qui représente $\log I$ en fonction du temps serait 92, 4 fois moindre; par conséquent, la valeur de $\lambda$ est restée inaltérée à la précision de $1 / 30000 \mathrm{Si}$, parmi les points expérimentaux, assez nombreux avant comme après l'exposition, on utilise les plus distants de part et d'autre de la droite moyenne, on trouve, comme limite extrême de la variation 1/2500, quantité certainement non atteinte.

2. Comparaison du rayonnement $\gamma$ d'ampoules de radium dont l'une est protégée par du plomb et l'autre exposée aux rayons solaires. - Cette expérience a été motivée par des publications signalant une influence notable des rayons solaires. sur l'activité du radium et de l'uranium; sans rechercher une précision supérieureà 
quelques millièmes, on a déterminé avec soin le rapport $R$ du rayonnement $\gamma$ de deux ampoules, contenant respectivement 5,4 et $8,0 \mathrm{mg}$ de radium. Ensuite la première de ces ampoules a été placée dans un tube de laiton introduit dans un bloc de plomb épais:l'autre a été mise dans un tube de celluloïd fixé à une planchette suspendue au-dessus du toit du Laboratoire et exposée au soleil. L'exposition a eu lıeu pendant 8 jours en juin. Après l'exposition, on a fait une nouvelle détermination du rapport $R$ qui a été trouvé le même qu'avant, à la précision de $\breve{a}$ pour mille au moins.

3. Expériences faites avec des lamelles portant une forte quantité de polonium. Une lamelle d'argent $\mathrm{L}$ avait reçu un dépôt de polonium formant une tache de $12 \mathrm{~mm}$ de diamètre, d'aspect noiràtre; le rayonnement $x$ émis produisait un courant d'ionisation évalué aussitôt après la préparation à $20000 \mathrm{E}$. S. environ. Le rayonnement pénátrant provenant de la présence de traces de $\mathrm{RaD}+\mathrm{RaE}$ était très faible; en plaçant la lame au-dessous d'une chambre à rayons $\beta$, de type courant, à $\breve{c m}$ de la feuille de base en aluminium très mince, on obtenait un courant d'environ $0,02 \mathrm{E}$. S. composé principalement de rayons $\beta$ et égal à la deux millième partie environ de celui qui correspondrait à l'équilibre entre la quantité de polonium considérée et $\mathrm{RaE}$ en présence de RaD. Les rayons $\beta$ étant déviés par un champ magnétique, il subsistait un faible rayonnement $\gamma$ attribuable au polonium, soit comme effet primaire, soit comme effet secondaire excité dans le support par les rayons $\alpha$, et ayant un coefficient d'absorption massique 240 dans l'aluminium, d'accord avec les données de Russell et Chadwick. La lame L produisait autour d'elle de l'ozone et altérait rapidement le papier soumis à son rayonnement.

a) On a soumis la lame $\mathrm{L}$ à un rayonnement $\beta$ intense, pour léceler une réversibilité 'éventuelle de la transformation de $\mathrm{RaE}$ en $\mathrm{Po}$; s'il se reformait du RaE, le rayonnement $\beta$ de la lame subirait une augmentation. On a donc comparé, aussi exactement que possible, le rayonnement $\beta$ de la lame $L$ à celui d'un étalon formé par une petite quantité de $R a D$ en équilibre avec $\mathrm{RaE}$ et Po. Le rapport trouvé 1,122 a été le même avant et après l'exposition aux rayons $\beta$. Celle-ci a duré 12 jours et a consisté à placer la lame $\mathrm{L}$ entre deux couches de tubes de radium, chaque couche étant formée de 5 tubes juxtaposés, contenant chacun $100 \mathrm{mgr}$ de radium. Admettant une précision de 1 pour 100 sur la mesure du rapport, on peut dire que l'exposition n'a pas produit une quantité de RaE de l'ordre de $\breve{5} \times 10^{-6}$ de celle qui serait en équilibre avec le polonium de la lame L en présence de RaD. Remarquons que le rayounement $\gamma$ qui constituait environ 10 pour 100 du rayonnement $\beta+\gamma$ de la lame $L$ a décru pendant l'exposition à peu près comme le polonium.

Une expérience semblable, mais plus précise, a été effectuée avec une autre lame $\mathbf{L}^{\prime}$ portant une quantité de polonium qui produisait un courant d'ionisation d'environ 12000 E.S. et occupait sur la lame une surface de $1 \mathrm{~cm}$ de diamètre. La purification étant meilleure, le rayonnement pénétrant $\beta$ était beaucoup plus faible que dans le cas précédent. Les mesures ont été faites par comparaison avec un étalon, soit avec un électroscope, soit avec un compteur à pointe de Geiger. L'exposition au rayonnement du radium a été faite dans les mèmes conditions que pour la lame L. Le rayonnement $\beta$ mesuré après l'exposition paraissait accuser une augmentation par rapport à celui mesuré avant l'exposition, mais l'effet ne m'a pas paru assez certain pour en tirer une conclusion favorable à l'existence du phénomène cherché.

Signalons, en outre, que sur la lame L on a observé, après la préparation, des variations de rayonnement pénétrant qui ont paru tout à fait anormales. Le rayonnement initial a d'abord décru pendant quinze jours jusqu'à une valeur insigniliante, mais a augmenté à nouveau ensuite pour atteindre une valeur triple de la valeur initiale, qui est restée fixe pendant quelques mois. L'allure de la variation n'a pu être expliquée.

b) La lame L a été utilisée pour irradier par son rayonnement $\alpha$ des préparations de $\mathrm{RaD}+\mathrm{RaE}$. Celles-ci étaient constituées par des lamelles en verre mince sur lesquelles on avait évaporé une goutte de solution contenant la matière active. Chaque lamelle était recouverte de deux feuilles d'aluminium battu très minces, l'enveloppant entièrement, puis fixée sur un disque de laiton; le rayonnement pénétrant de chaque lamelle était ensuite 
mesuré aussi exactement que possible. Puis l'une des lamelles était exposée aux rayons $a$ issus de la lame $\mathrm{L}$ posée, avec la face active tournée vers le bas, sur un disque de papier percé d'une fenètre et reposant sur la lamelle à irradier. Les rayons a passant par la fenêtre traversaient la double feuille d'aluminium qui diminuait à peine leur parcours, mais les empêchait d'arracher des parcelles de matière active rencontrée. On a fait deux expositions successives, de 5 jours chacune, alternant avec trois séries de mesures. Le rayonnement pénétrant des deux lamelles est resté constant, et il en a été de même pour le rapport, à la précision des mesures qui était de l'ordre du millième.

4. Expériences sur la formation de $R a E$ et $P_{0}$ à partir des solutions de $R a D$. - La préparation et la manipulation de solutions de RaD et de ses dérivés à partir des ampoules de radon, après destruction de celui-ci, devenue d'usage courant au Laboratoire Curie (1), a donné lieu à quelques incidents, jusqu'ici inexpliqués, qui suggéraient des anomalies dans les transformations radioactives de la matière actıve concentrée. Quelques recherches approfondies entreprises pour cette raison n'ont cependant pas confirmé l'existence ds ces anomalies.

La matière soumise à l'expérience était composée de quelqnes milligrammes de plomb $\left(^{2}\right)$ contenant environ $0,2 \mathrm{mg}$ de $\mathrm{RaD}$; conservée à sec dans un creuset de quartz, elle attaquait celui-ci, produisant généralement de petits trous pareils à des piqûres d'épingles et parfois des fèlures. A l'état de nitrate ce plomb actif subissait une réaction qui Iui faisait prendre une couleur brune; à la dissolution dans $\mathrm{HAzO}^{3}$ dilué, on voyait alors apparaître des paillettes noires de peroxyde qu'on retransformait en nitrate par digestion avec de l'acide plus concentré.

L'accroissement de Po (radium F) à partir de RaD privé de ses dérivés a lieu suivant une formule déduite de la théorie des transformations radioactives. Soient respectivement $\lambda_{1}, \lambda_{2}, \lambda_{3}$ les constantes radioactives des corps $\mathrm{D}$, E et $\mathrm{F}$, ces mêmes lettres désignant les nombres d'atomes des substances. Négligeant $\lambda_{1}$ par rapport à $\lambda_{2}$ et prenant

on trouve

$$
\lambda_{1}=0,0433 \mathrm{an}^{-1} ; \lambda_{2}=52,3 \mathrm{an}^{-1} ; \lambda_{3}=1, \times 14 \mathrm{an}^{-1}
$$

$$
F=0,0244 D_{0}\left[\mathrm{e}^{-\lambda_{1} t}+0.035 \mathrm{e}^{-\lambda_{2} t}-1,035 \mathrm{e}^{-\lambda_{3} t}\right] .
$$

Pour $t>0,1$ an, cette formule se réduit à

$$
F^{\prime} \doteq 0,0244 D_{0}\left[\mathrm{e}^{-\lambda_{1} t}-1,03 \ddot{i} \mathrm{e}^{-\lambda_{3} t}\right] .
$$

La valeur maximum de $\mathrm{F}$, atteinte en 777 jours est $0,89 \times 0,0244 \mathrm{D}_{0}$.

L'accroissement de RaE a lieu suivant une loi plus simple. Négligeant $\lambda_{1}$ devant $\lambda_{2}$, on trouve

$$
E=\frac{\lambda_{1} D_{0}}{\lambda_{2}}\left(\mathbf{e}^{-\lambda_{1} t}-\mathbf{e}^{-\lambda_{2} t}\right)
$$

formule qui se réduit à son premier terme pour $t>1$ mois.

Le premier essai a consisté à vérifier que l'accumulation de RaE a lieu de la même manière dans le sel solide et dans sa solution. Pour cela, le rayonnement $\beta$ du sel solide a été mesuré avec soin dans des conditions déterminées; le sè a été ensuite dissous et laissé en solution pendant 13 jours, puis la solution a été évaporée el le rayonnement $\beta$ du résidu a été mesuré à nouveau dans des conditions aussi semblables que possible à celles des mesures précédentes. Aucune différence systématique n'a été observée entre les deux séries de mesures. Le courant d'ionisation mesuré dans une chambre à rayons $\beta$ avait une valeur d'environ $3,5 \mathrm{E}$. S. Le courant d'ionisation relatif au polonium en équilibre avec le RaD correspondant devait ètre environ 35000 E. S.

(') Irène Curie, J. Chim. phys., t. 22 (1925), p. 471.

(2) La solution du dépôt actif extrait du verre pilé contenait du plomb provenant du verre des a npoules ou du mercure. 
On a laissé ensuite le polonium s'accumuler dans le sel solide pendant 178 jours, après quoi il a été procédé à la dissolution du sel et au dosage de la solution $\mathrm{S}$ par gouttes. Cette méthode de dosage a été décrite en détail dans une publication précédente $\left(^{1}\right)$. Le dosage a porté sur le polonium (rayons $\alpha$ ) et sur le RaE (rayons $\beta$ ). Les lamelles d'épreuve portant des petites fractions de la solution évaporées, ont été conservées et l'accroissement de leur rayonnement a élé suivi à titre de contrôle. Après le dosage la solution $S$ a été évaporée à sec, sauf une iraction représentée par l'une des solutions' secondaires qui avaient servi au dosage, soit la solution $\mathrm{F}$ contenant $0, \breve{5}$ pour 100 de la matière totale. On a laissé se poursuivre à nouveau l'accumulation de Po dans le sel solide pendant 102 jours, consécutifs aux 18 jours où le sel était resté dissous pendant les opérations de dosage. Après cette deuxième période d'accumulation, le sel a été remis en solution, et il a été fait un nouveau dosage par la méthode des gnuttes. Par ailleurs, un dosage a été fait aussi sur la solution $\mathrm{F}$ qui avait été conservée inaltérée. Toutes ces opérations ont donné des résultats concordants; à la précision des mesures qui était de l'ordre de $0, \mathfrak{5}$ pour 100 à 1 pour 100 , on a trouvé le mème accroissement relatif de polonium dans le sel de plomb solide à activité concentrée, dans la matière solide très peu concentrée portée par les lamelles servant de témoin et dans la solution diluée $\mathrm{F}$.

Deux mois et demi environ après, ces résultats ont été confirmés par l'extraction du polonium formé dans la solution $S$, et par son dosage. L'extraction a été faite à l'aide de lames d'argent qu'on a fait tourner dans la solution faiblement nitrique contenant aussi une trace d'acide chlorhydrique. Dans la solution restante, on a dosé le rayonnement $\alpha$ et le rayonnement $\beta$. Les lames d'argent ont été dissoutes pour séparer l'argent, et sur la solution contenant le polonium on a fait un dosage. Le polonium extrait correspond à $31450 \mathrm{E}$. S., celui non extrait à $600 \mathrm{E}$. S. Le radium E n'a pas été extrait et correspond à $230 \mathrm{E}$. S., cette valeur étant relative à la chambre d'ionisation pour mesure de rayons $x$ dans laquelle l'efficacité du radium $\mathbf{E}$ en équilibre avec le polonium en présence de $\mathrm{RaB}$ était environ 1 pour 100 de celle du polonium. On voit que l'extraction du polonium a été faite à 2 pour 100 près. La quantité totale de polonium mise en évidence par le dosage est en accord avec les prévisions déduites du rayonnement des lamelles témoins.

L'ensemble des résultats se laisse représenter par la formule théorique ci-dessus donnée, àvec les valeurs numériques, en unités E. S. :

$$
I_{{ }_{\alpha}}=38600\left(\mathbf{e}^{-i_{1} t}-1,03 \check{\mathbf{e}^{-i_{3} t}}\right)
$$

$I x$ étant le courant d'ionisation dû aux rayons $\alpha$ du polonium formé dans la solution $\mathrm{S}$.

Toutefois, les extractions postérieures ont montré une certaine divergence avec cette formule, et il y a lieu de se demander si la période du RaD n'est pas un peu plus grande que la valeur admise $\left({ }^{2}\right)$.

En relation avec ces expériences, rappelons que l'accroissement du débit de chaleur d'une ampoule de radium par formation de polonium, pendant dix-sept ans, s'est montré conforme à la théorie, ainsi qu'il résulte d'une publication antérieure $\left(^{3}\right)$.

5. Exposition de lamelles portant un dépôt de polonium au rayonnement pénétrant du radium. -- Il a été fait à ce sujet quatre expériences. Dans la première, on a utilisé deux lamelles d'argent portan' chacune en son milieu un dépôt de polonium invisible occupant une surface de quelrues $\mathrm{mm}$ de diamètre. Les lamelles ont été placées dans des chambres d'ionisalion $\alpha$ de même type, où leur rayonnement $\alpha$ était complètement absorbé. On a mesuré à l'aide d'un électromètre et d'un quartz piézo électrique, le courant de saturation $I$ ou $I^{\prime}$ produit par chaque lamelle avec une tension de mème signe sur la chambre. Puis on établissait sur les chambres des tensions de signe contraire et on mesurait la différence $i_{1}$ des

(') Mme Curie, J. Chim. phys., t. 22 (1925), p. 142.

$\left({ }^{2}\right)$ Cette présomption parait confirmée par des expériences qui seront publiées ultérieurement.

(3) Mme Curir et D.-K. Yovanovitch, J. Phys., t. 6 (1925), p. 33. 
courants de saturation; enfin, on renversait le sens des tensions et on mesurait la différence $i_{2}$, à peu près égale à - $i_{1}$. Dans toutes ces mesures, on tenait compte du mouvement propre de l'appareil. Chaque courant était de l'ordre d'une unité E. S., au début des mesures, et la différence $i$ de l'ordre de 0,1 E. S. Les mesures étaient poursuivies pendant quinze jours, puis on a procédé à l'exposition d'une des lamelles. L'exposition a été faite de la manière suivante: sur la lamelle d'argent portant le polonium on plaçait une lamelle de verre mince protectrice fixée aux bords; on installait le tout, entre deux couches de tubes de radium, dans une boîte. On a pu utiliser à cet effel 10 tubes de radium contenant chacun $100 \mathrm{mg}$ de $\mathrm{Ra}, 8$ autres tubes contenant ensemble $200 \mathrm{mg}$ de Ra et une ampoule contenant $300 \mathrm{mg}$ de Ra. Les tubes étaient disposés de la manière la plus favorable, en égard à leurs dimensions et à celles de la région irradiée, qui recevait leur rayonnement $\beta$ et $\gamma$ ayant traversé le verre des ampoules. La durée de l'exposition a été de 67 jours. Après l'exposition, on a libéré la lamelle exposée de la lamelle protectrice en verre et on a mesuré avec soin l'activité acquise par celle-ci par contact avec le polonium, et dont il a été tenu compte. La lamelle non exposée a été conservée dans une boîte sous une feuille de papier maintenue à très petite distance de sa face active; cette feuille a été trouvée inactive quand les mesures ont été reprises. La série de mesures flnales après lexposition a été poursuivie pendant quelques jours, selon les mèmes modalités que la série initiale. Chacune de ces séries permet de définir un point moyen initial et un point moyen final qui servent de base pour le calcul de la constante radioactive.

Cette manière d'opérer a paru indispensable du fail que les mesures ne présentent pas la régularité qu'on devrait en espérer. Bien que, dans une série déterminée, la distribution des écarts soit à peu près conforme aux prévisions basées sur la théorie des Aluctuations, les moyennes obtenues dans les séries faites à un ou plusieurs jours d'intervalle s'écartent parfois des droites moyennes qui représentent $\log I$ ou $\log i$ en fonction du temps, plus que ne le fait prévoir la précision des mesures. Ce manque de régularité est probablement attribuable à divers effets de faible importance produisant une absorption non négligeable sur les rayons $\alpha$.

Dans l'expérience 1 la décroissance de $\log I$ et de $\log I^{\prime}$ a été très exactement linéaire, toutes corrections faites, selon la période de 140,0 jonrs. La pente de la droite représentative de Log $i$ dıffère de la pente normale d'environ 6 pour cent, ce qui correspondrait à une augmentation de $\lambda$ de 0.6 pour 100 pour la lamclle exposée. D'autres expériences paraissaient nécessaires.

On a apporté le plus de soins possible à examiner les conditions de ces nouvelles expériences. On a vérifié que la saturation du courant était atteinte à une précision suffisante. On a employé du polonium très pur, et les lamelles étaient toujours protégées dans l'intervalle des mesures par des feuilles minces d'aluminium ou de mica dont on contrôlait l'activité pour en tenir compte. On a vérifié que le contact des tubes de radium ne produisait pas d'activation appréciable. Les mesures étaient faites, par séries de 10, le temps de compensation ( 30 secondes à une minute ou un peu plus), était modifié de manière à faire porter les mesures sur des nombres peu différents de rayons.

Dans l'expérience 2 on a utilisé 4 lamelles en argent portant chacune à l'origine une quantité de polonium correspondant à 0,6 E. S. La lamelle IV était considérée comme étalon, et l'on mesurait son intensité $I_{4}$ ainsi que l'excès des intensités $I_{1}, I_{2}, I_{3}$ des autres lamelles sur $I_{4}$, qui était de l'ordre de 10 pour cent de $I_{4}$. Les lamelles I et III ont été exposées et la lamelle II servait de témoin. Pendant l'exposition qui a duré 174 jours, la lamelle I était placée entre deux séries de tubes de radium et la lamelle III, placée en-dessus, recevait le rayonnement d'un seul côté. La précision des mesures était environ 0,3 pour cent pour $I_{4}$ et environ 1 pour 100 pour les différences. Les écrans protecteurs en mica sont restés inactifs. La valeur de $\lambda$ relative à la décroissance de $I_{+}$est $\curlywedge=0,00500$ jour ${ }^{-1}$ (période 138,6 jours). Le rapport $\frac{I_{2}-I_{4}}{I_{4}}$, avant et après exposition, est resté le même à 0,2 pour cent près. tandis que le rapport $\frac{I_{1}-I_{4}}{I_{4}}$ a diminué de 5,2 pour cent et le 
rapport $\frac{I_{3}-I_{4}}{l_{4}}$ de 3 pour cent. Il paraissait donc y avoir une augmentation de la vitesse de destruction pour les lamelles exposées, la constante $\lambda$ étant remplacée par $\lambda(1+\xi)$; la variation relative $\varepsilon$ se calcule par la relation

$$
\lambda \varepsilon t=\frac{I_{0}^{\prime}-I_{0}}{I_{0}^{\prime}}-\frac{i \mathbf{e}^{\lambda t}}{I_{0}^{\prime}}
$$

on aurait dans le cas actuel pour $\_$environ $\mathbf{4}, 5$ millièmes pour la lamelle 1 et 4 millièmes pour la lamelle III. Un tel effet serait relativement considérable, et devait être mis en doute. La période obtenue pour l'étalon IV étant inférieure de 0,7 pour 100 à celle qu'on admet au Laboratoire Curie ( 139,5 jours), on pouvait penser qu'il $y$ avait eu un faible effet de pénétration de polonium dans l'argent pour la lamelle IV, et que cet effet avait été accentué pour les lamelle I et III par l'action du rayonnement même par l'échauffement local produit dans la boîte fermée qui avait contenu les tubes de radium et les lamelles.

On a done fait une troisième expérience avec du polonium déposé sur des lamelles de verre, par évaporation de gouttes de solution diluée. Cependant, le verre qui est préfépable à l'argent au point de vue de la pénétration, est un support moins favorable que celui-ci en ce que le dépôt du polonium est moins bien formé. Dans le cas de Ag, on enlève le liquide qui contenait le polonium, après l'abandon de la matière active; celle-ci adhère au support et on vérifie par photographie qu'elle est distribuée en tache uniforme bien limitée. Dans le cas du verre, l'évaporation laisse le polonium concentré sur un anneau irrégulier, avec adhérence médiocre, et cela d'autant plus que le verre a été plus soigneusement nettoyé. La régularité des mesures est moindre avec le verre qu'avec l'argent. On a préparé 6 lamelles de verre dont la plus active (IV) a été choisie comme étalon et la lamelle I comme témoin; les lamelles III et VI ont été exposées; les lamelles II et V ont été emballées dans une boîte et envoyées en Belgique à l'usine de radium et d'urane d'Oolen, où la boîte a été profondément enfoncée dans un tas d'uranate de soude, dans lequel elle a séjourné 6 mois. Au retour de la boìte à Paris, on a constaté que, malgrế les soins apportés à l'emballage et au transport, la distributlon de matière active avait été altérée; le résultat de l'exposition, en ce cas, est donc resté inconnu. L'exposition des lamelles III et VI au rayonnement du radium a été faite pendant 130 jours dans des conditions semblables à celles de la deuxième expérience. Après l'exposition, on a constaté qu'une petite quantité de polonium avait passé sur les écrans protecteurs. D'après l'ensemble des points représentatifs des mesures avant et après l'exposition, et toutes corrections faites, on a estimé que la décroissance de l'étalon IV et de la lamelle témoin I correspondait à une période de $13 \%,:$ jours inférieure de $1, \ddot{3}$ pour cent à la période admise comme normale et que la décroissance des lamelles exposées paraissait ici, encore accélérée à peu près comme dans l'expérience 2 , avec une valeur de six millièmes pour $\varepsilon$.

Puisque les lamelles avaient perdu une petite fraction de leur polonium, par projection ou par manque d'adhérence, on a procédé à une quatrième expérience avec un support différent. On a choisi une couche mince de nickel déposée sur du verre par pulvérisation cathodique, On espérait ainsi obtenir une bonne adhérence avec peu de chance de pénétration. Les couches de nickel étaient transparentes, leur couleur variait avec l'épaisseur du brun pâle au brun plus foncé; leur épaisseur a été sommairement évaluée par des pesées et des comparaisons de teinte. Elles étaient uniformes et très adhérentes au verre. Le polonium a été déposé sur chaque lamelle d'une goutte de solution acétique très acide. Sur une couche de nickel très mince, teinte jaune pàle, épaisseur présumée inférieure à $10 \mu \mu$, le polonium ne se déposait pas; sur une telle couche on ne pouvait pas non plus constater l'existence de la conductibilité électrique métallique $\left(^{1}\right)$. Les couches d'environ $20 \mu$ utilisées dans l'expérience permettent un excellent dépôt invisible de polonium: leur conductibilité électrique est prononcée quoique inférieure à la conductibilité normale du nickel compact. On a préparé 4 lamelles dont les plages actives donnaient des images photographiques

(1) Tout au moins avec les prises de contacl employées qu'on n'a pas cherché à rendre très parfaites. 
uniformes et bien limitées. La lamelle IV, la plus active, a été prise comme étalon, les lamelles I et III ont été exposées, la lamelle II a servi de témoin. Après l'exposition qui a duré 182 jours, toutes les lamelles ont paru inaltérées. L'intensité initiale était environ 1 E. S. et les différences ら pour 100 de cette valeur. De l'ensemble des mesures qui n'ont pas été aussi satisfaisantes qu'on l'avait espéré, on a conclu que la loi de décroissance de la lamelle III ne présentait pas de différence appréciable avec celle de la lamelle témoin et que pour la lamelle I, il existait une petite différence conduisant à la valeur $\approx=1 / 2000$, environ 10 fois plus petite que dans les expériences précédentes. Ainsi il est vraisemblable que les résultats de ses dernières sont attribuables à des effets parasites, tels que la pénétration du polonium dans le support, son déplacement à la surface du support ou sa projection en dehors de celui-ci. On voit, en même temps, à quel genre de difficultés se heurtent l'exécu tion et l'interprétation d'essais semblables.

Il pout être utile de donner, à titre d'exemple, l'examen d'une série de mesures au point de vue des fluctuations radioactives. Dans la compensation par un poids agissant sur un quartz piezoélectrique, la quantité d'électricité apportée à l'électromètre dans une mesure reste constante et de même le nombre de particules nécessaire pour la libérer; c'est donc l'intervalle de temps qui est soumis à la fluctuation. On a reporté celle-ci sur le nombre de particules, en admettant qu'en première approximation à une petite augmentation $\tau$ de l'intervalle moyen $\Theta$ correspond une diminution $Z \tau / \Theta$ du nombre $Z$ de particules relatives à l'intervalle moyen $\Theta$. On trouve alors pour la valeur la plus probable de $\Sigma \tau^{2}$ dans une série de mesures du courant principal $\Sigma \mathbf{z}^{2}=\frac{(m-1) \Theta^{2}}{Z}$, et pour une série de mesures de la différence

$$
\mathbf{\Sigma}_{-2}^{2}=(m-1) \Theta^{2} \frac{\left(Z+Z^{\prime}\right)}{\left(Z-Z^{\prime}\right)^{2}} .
$$

Dans un exemple, où un nombre $Z$ de particules était reçu par intervalle moyen de $19^{\prime \prime}, 5^{\bullet}$, défini par une série de 10 mesures faites sur le courant principal, on pouvait prévoir $\Sigma \mathbf{-}^{2}=0,011 ;$ on a trouvé 0,03 . Dans la mesure consécutive d'une différence, $2,04 \times 10^{4}$ particules étaient reçues en un intervalle moyen $37^{\prime \prime}, 1$ défini par une série de 10 mesures; on avait $Z+Z^{\prime}=5,98 \times 10^{3}$; on pouvait prévoir $\Sigma_{\tau^{2}}=17,8$, on a trouvé $\mathbf{1 0 , 8}$. Une autre série de mesures de la mème différence a donné un intervalle moyen $36^{\prime \prime}, 12$; la valeur de $\Sigma \tau^{2}$ trouvée 17,ă était très voisine de la valeur 17,0 prévue. Ces exemples montrent que les écarts des mesures d'une série étaient de l'ordre de ceux que doit produire la fluctuation sur l'émission de rayons $\alpha$. Les écarts entre les moyennes successives doivent être attribués à des effets d'une autre nature qui jouent un rôle beaucoup plus important dans la mesure de rayons $\alpha$ que dans celle de rayons $\gamma$.

L'ensemble des expériences ici décrites est à l'appui de toutes celles déjà .publiées par de nombreux auteurs, qui n'ont pas réussi à mettre en évidence des écarts des lois de transformation radioactives. Un certain nombre de détails relatifs à ces expériences peuvent, je crois, être de quelque utilité pour l'examen de cette question, et c'est là un des buts de cette publication.

Les nombreuses manipulations et mesures indispensables pour ces expériences ont exigé le concours de plusieurs collaborateurs, et il m'est agréable de remercier pour leur assistance $\mathbf{M}^{\mathrm{me}}$ Irène Curie, $\mathbf{M}^{\text {1les }}$ Galabert et Maracineanu, M. Cailliet et M. Joliot. 